\title{
Two parameter Ridge estimator in the inverse Gaussian regression model
}

\author{
Y. Murat Bulut*1 (D), Melike Işlar² (iD) \\ ${ }^{1}$ Eskişehir Osmangazi University, Faculty of Science and Letters, Department of Statistics, 26040, \\ Eskişehir, Turkey \\ ${ }^{2}$ Eskişehir Osmangazi University, Graduate School of Natural and Applied Sciences, 26040, Eskişehir, \\ Turkey
}

\begin{abstract}
It is well known that multicollinearity, which occurs among the explanatory variables, has adverse effects on the maximum likelihood estimator in the inverse Gaussian regression model. Biased estimators are proposed to cope with the multicollinearity problem in the inverse Gaussian regression model. The main interest of this article is to introduce a new biased estimator. Also, we compare newly proposed estimator with the other estimators given in the literature. We conduct a Monte Carlo simulation and provide a real data example to illustrate the performance of the proposed estimator over the maximum likelihood and Ridge estimators. As a result of the simulation study and real data example, the newly proposed estimator is superior to the other estimators used in this study.
\end{abstract}

Mathematics Subject Classification (2020). 62J07, 62J12

Keywords. inverse Gaussian regression, biased estimators, two parameter Ridge estimator, multicollinearity

\section{Introduction}

In many real-life problems of the regression model, the distribution of the response variable may not be normal. In this situation, the linear regression model (LRM) does not be used. Therefore, generalized linear models (GLMs) are used to model data set in which the distribution of the response variable is different from the normal distribution. If the response variable is positively skewed and continuous, then the distribution of the response variable may be inverse Gaussian (IG) distribution. The probability density function (pdf) of the IG distribution is

$$
f(y ; \mu, \tau)=\frac{1}{\sqrt{2 \pi y^{3} \tau}} \exp \left[-\frac{1}{2 y}\left(\frac{y-\mu}{\mu \sqrt{\tau}}\right)^{2}\right], y>0, \mu>0, \tau>0,
$$

\footnotetext{
*Corresponding Author.

Email addresses: ymbulut@ogu.edu.tr (Y.M. Bulut), melikeisilar@gmail.com (M. Işılar)

Received: 20.10.2020; Accepted: 28.02.2021
} 
where $\mu$ and $\tau$ are location and scale parameters, respectively, also, mean and variance of the IG distribution can be obtained as $\mu$ and $\tau \mu^{3}$. For more details about the IG distribution and applications, see [8,18,19,24].

Because the IG distribution is a member of the exponential family, the inverse Gaussian regression (IGR) model is a particular form of the GLMs. Generally, the maximum likelihood (ML) estimation method is used to estimate the unknown coefficients of the IGR model. But, if the explanatory variables are highly collinear that is known as the multicollinearity problem, the ML estimation is affected negatively. As a result of the multicollinearity problem, the wrong sign of the estimated coefficients can be obtained, the confidence interval of the ML estimator is getting wider. Also, the scaler mean square error (MSE) value is getting bigger.

Several methods have been proposed to handle the multicollinearity problem. One of the most popular ways is the Ridge regression method, developed by [11], which is very helpful in many regression applications. Algamal [2] has proposed the Ridge estimator (RE) in the IGR model. The main advantage of the RE is that the MSE value is less than that of the ML estimator. But, on the other hand, RE has some disadvantages. For example, $\mathrm{RE}$ does not improve the quality of fit of a regular regression. Also, RE can not be interpreted because the properties of the RE are similar to the nonlinear regression model. Huang and Yang [12] generalized the Ridge and Liu estimators in the negative binomial regression model (NBRM) which is a special case of GLMs. Then, Amin et al. [4] investigated the performance of two parameter estimator in the gamma regression model (GRM). Also, Toker et al. [22] developed first order two parameter estimator in the GLMs.

Then, after the Ridge estimator proposed for the IGR model, some works have been done for the IGR model. Some of them are given as follows. Amin et al.[3] proposed some biasing parameter estimators for the RE in the IGR model, Akram et al. [1] introduced the Liu estimator and two parameter estimator, which is defined by [17], for the IGR model, Naveed et al. [16] introduced a new shrinkage paremeters for the inverse Gaussian Liu estimator.

Because of the disadvantages of the RE, Lipovetsky and Conklin [14] and Lipovetsky [13] have proposed a generalization of the $\mathrm{RE}$ that is called two-parameter Ridge estimator (TPRE). TPRE always outperforms than RE and ML estimator, and has some excellent properties of orthogonality between residuals and predicted values on the dependent variable. The second parameter in the TPRE improved the quality of fit of the regression model. In the LRM, Toker and Kaçıranlar [21] compare the TPRE with ordinary least squares (OLS) estimator and RE via matrix mean square error (MMSE) criteria. In the GLMs framework, TPRE has been used in the binary logistic regression model by [6].

In this paper, Section 2 describes the IGR model and estimation method of the IGR model. In Section 3, we introduce TPRE in the IGR model. We compare the proposed estimator with the RE and ML estimator in Section 4. In Section 5, algorithms are given to select $k$ and $q$ parameters. Monte Carlo simulation study and a real data example are given to show the usefulness of the proposed estimator in Sections 6 and 7, respectively. Finally, in Section 8, concluding remarks are given.

\section{Methodology}

Because the IG disribution is the member of the exponential family, the Eq. (1.1) can be rewritten as

$$
f(y ; \mu, \tau)=\exp \left\{-\frac{y}{2 \mu^{2} \tau}+\frac{1}{\mu \tau}-\frac{1}{2 y \tau}-\frac{1}{2} \ln \left(2 \pi y^{3}\right)-\frac{1}{2} \ln \tau\right\} .
$$

Using the Eq. (2.1), the log-likelihood function of the IG distribution is obtained as 


$$
\ell(\mu, \tau)=\sum_{i=1}^{n}\left[-\frac{y_{i}}{2 \mu^{2} \tau}+\frac{1}{\mu \tau}-\frac{1}{2 y_{i} \tau}-\frac{1}{2} \ln \left(2 \pi y_{i}^{3}\right)-\frac{1}{2} \ln \tau\right] .
$$
as

If we use the canonical link function $\mu_{i}=\frac{1}{\sqrt{x_{i}^{T} \beta}}$, the log-likelihood function is rewritten

$$
\ell\left(\mu_{i}, \tau\right)=\sum_{i=1}^{n}\left[-\frac{y_{i} x_{i}^{T} \beta}{2 \tau}+\frac{\sqrt{x_{i}^{T} \beta}}{\tau}-\frac{1}{2 y_{i} \tau}-\frac{1}{2} \ln \left(2 \pi y_{i}^{3}\right)-\frac{1}{2} \ln \tau\right],
$$

where $\tau$ is the dispersion parameter. The estimated dispersion parameter can be obtained as

$$
\widehat{\tau}=\frac{1}{n-p-1} \sum_{i=1}^{n} \frac{\left(y_{i}-\widehat{\mu}_{i}\right)^{2}}{\widehat{\mu}_{i}^{3}} .
$$

Taking the first derivative of the log-likelihood function then setting them to zero, we obtain the following non-linear equation

$$
U(\beta)=\frac{1}{2 \tau} \sum_{i=1}^{n}\left[y_{i}-\frac{1}{\sqrt{x_{i}^{T} \beta}}\right] x_{i}=0, \quad i=1,2, \ldots, n .
$$

Since Eq. (2.5) is a non-linear function of the parameter vector $\beta$, iterative methods like iteratively reweighted least squares (IRLS) must be used to estimate unknown parameters. In each iteration of the IRLS, the parameters are obtained as

$$
\beta^{(r)}=\beta^{(r-1)}+I^{-1}\left(\beta^{(r-1)}\right) U\left(\beta^{(r-1)}\right),
$$

where $U\left(\beta^{(r-1)}\right)$ and $I\left(\beta^{(r-1)}\right)=\left[-E\left(\frac{\partial \ell\left(\mu_{i}, \tau\right)}{\partial \beta \partial \beta^{T}}\right)\right]$ are the score vector, and the Fisher information matrix evaluated at $\beta^{(r-1)}$, respectively. When convergence holds, the final step of the estimated coefficients is defined as

$$
\widehat{\beta}_{M L}=\left(X^{T} \widehat{W} X\right)^{-1} X^{T} \widehat{W} \widehat{z},
$$

where $\widehat{z}_{i}=\widehat{\eta}_{i}+\frac{\left(y_{i}-\widehat{\mu}_{i}\right)}{\widehat{\mu}_{i}^{3}}$ and $\widehat{W}=\operatorname{diag}\left(\widehat{\mu}_{i}^{3}\right), i=1, \ldots, n$, are adjusted response variable and weight matrix, respectively. The mean vector and covariance matrix of the ML estimator are given as

$$
\begin{gathered}
E\left(\widehat{\beta}_{M L}\right)=\beta, \\
\operatorname{Var}\left(\widehat{\beta}_{M L}\right)=\tau\left(X^{T} \widehat{W} X\right)^{-1} .
\end{gathered}
$$

The matrix mean square error (MMSE) and scaler mean square error (MSE) of the ML estimator are given as follows

$$
\operatorname{MMSE}\left(\widehat{\beta}_{M L}\right)=\tau C^{-1}
$$

where $C=X^{T} \widehat{W} X$.

$$
\begin{aligned}
\operatorname{MSE}\left(\widehat{\beta}_{M L}\right) & =\operatorname{tr}\left[M M S E\left(\widehat{\beta}_{M L}\right)\right] \\
& =\tau \sum_{j=1}^{p+1} \frac{1}{\lambda_{j}},
\end{aligned}
$$

where $\operatorname{tr}(\cdot)$ is trace operator and $\lambda_{j}$ is the $j^{\text {th }}$ eigenvalue of the $X^{T} \widehat{W} X$ matrix such that $\lambda_{1} \geq \lambda_{2} \geq \cdots \geq \lambda_{p+1}$. 
When the multicollinearity exists among the explanatory variables, variance of the ML estimator inflates. In this situation, following Ridge estimator has been proposed.

\subsection{Inverse Gaussian Ridge estimator}

Ridge estimator is commonly used biased estimator to combat multicollinearity problem in LRM and GLMs. In IGR model, Algamal [2] proposed inverse Gaussian Ridge estimator (IGRE) to handle the multicolinearity problem. IGRE is given as

$$
\begin{aligned}
\widehat{\beta}_{I G R E}(k) & =\left(X^{T} \widehat{W} X+k I\right)^{-1} X^{T} \widehat{W} X \widehat{\beta}_{M L} \\
& =C_{k}^{-1} C \widehat{\beta}_{M L},
\end{aligned}
$$

where $k>0$ is the ridge parameter and $C_{k}^{-1}=\left(X^{T} \widehat{W} X+k I\right)^{-1}$. The covariance matrix and bias vector of the IGRE can be obtained as

$$
\begin{gathered}
\operatorname{Var}\left(\widehat{\beta}_{\text {IGRE }}(k)\right)=\tau C_{k}^{-1} C C_{k}^{-1}, \\
b_{\text {IGRE }}=\operatorname{Bias}\left(\widehat{\beta}_{\text {IGRE }}(k)\right)=-k C_{k}^{-1} \beta .
\end{gathered}
$$

The MMSE and scaler MSE of the IGRE are given as

$$
\begin{aligned}
\operatorname{MMSE}\left(\widehat{\beta}_{\text {IGRE }}(k)\right) & =\operatorname{Var}\left(\widehat{\beta}_{\text {IGRE }}(k)\right)+b_{\text {IGRE }} b_{\text {IGRE }}^{T} \\
& =\tau C_{k}^{-1} C C_{k}^{-1}+k^{2} C_{k}^{-1} \beta \beta^{T} C_{k}^{-1}, \\
\operatorname{MSE}\left(\widehat{\beta}_{\text {IGRE }}(k)\right) & =\operatorname{tr}\left[\operatorname{MMSE}\left(\widehat{\beta}_{\text {IGRE }}(k)\right)\right] \\
& =\tau \sum_{j=1}^{p+1} \frac{\lambda_{j}}{\left(\lambda_{j}+k\right)^{2}}+k^{2} \sum_{j=1}^{p+1} \frac{\alpha_{j}^{2}}{\left(\lambda_{j}+k\right)^{2}},
\end{aligned}
$$

where $\alpha_{j}$ is the $j^{\text {th }}$ element of the $Q^{T} \beta$ vector. The columns of the $Q$ matrix contain the eigenvectors of the $X^{T} \widehat{W} X$ matrix. To estimate the $k$ parameter, there are lots of methods, we refer the work of [3] for further details.

\section{Proposed estimator}

In this study, we extend a two-parameter Ridge estimator (TPRE) for the IGR model to cope with the multicollinearity problem. TPRE is proposed by [14] and [13] in the LRM. It is emphasized that TPRE is superior to Ridge and OLS estimators in the LRM, because of the parameter $q$. This parameter is related to the quality of fit. We hope that inverse Gaussian two parameter Ridge estimator (IGTPRE) will be superior over the IGRE and ML estimator. IGTPRE is defined as

$$
\widehat{\beta}_{\text {IGTPRE }}(k, q)=q(C+k I)^{-1} r,
$$

where $r=X^{T} \widehat{W} \widehat{z}$, and $q=\frac{r^{T}(C+k I)^{-1} r}{r^{T}(C+k I)^{-1} C(C+k I)^{-1} r}>0$.

Since the IGTPRE is the generalization of the IGRE and ML estimator, the IGRE and ML estimator can be obtained as special cases as follows

$$
\begin{gathered}
\widehat{\beta}_{\operatorname{IGTPRE}}(0,1)=\widehat{\beta}_{M L}, \\
\widehat{\beta}_{I G T P R E}(k, 1)=\widehat{\beta}_{I G R E}(k) .
\end{gathered}
$$


The bias vector and covariance matrix of the proposed estimator can be obtained as follows

$$
\begin{aligned}
b_{\text {IGTPRE }}=\operatorname{Bias}\left(\widehat{\beta}_{\text {IGTPRE }}(k, q)\right) & =E\left(\widehat{\beta}_{\text {IGTPRE }}\right)-\beta \\
& =\left[q C_{k}^{-1} C-I\right] \beta, \\
\operatorname{Var}\left(\widehat{\beta}_{\text {IGTPRE }}(k, q)\right) & =E\left[\left(\widehat{\beta}_{\text {IGTPRE }}(k, q)-\beta\right)\left(\widehat{\beta}_{\text {IGTPRE }}(k, q)-\beta\right)^{T}\right] \\
& =q^{2} \tau C_{k}^{-1} C C_{k}^{-1} .
\end{aligned}
$$

Using the bias and covariance, MMSE and scaler MSE are computed as

$$
\begin{aligned}
\operatorname{MMSE}\left(\widehat{\beta}_{\text {IGTPRE }}(k, q)\right) & =\operatorname{Var}\left(\widehat{\beta}_{\text {IGTPRE }}(k, q)\right)+b_{\text {IGTPRE }} b_{\text {IGTPRE }}^{T} \\
& =q^{2} \tau C_{k}^{-1} C C_{k}^{-1}+b_{\text {IGTPRE }} b_{\text {IGTPRE }}^{T}, \\
\operatorname{MSE}\left(\widehat{\beta}_{\text {IGTPRE }}(k, q)\right)= & \operatorname{tr}\left[\operatorname{MMSE}\left(\widehat{\beta}_{\text {IGTPRE }}(k, q)\right)\right] \\
= & \operatorname{tr}\left[\operatorname{Var}\left(\widehat{\beta}_{\text {IGTPRE }}(k, q)\right)\right]+b_{\text {IGTPRE }}^{T} b_{\text {IGTPRE }} \\
& =\sum_{j=1}^{p+1} \frac{q^{2} \tau \lambda_{j}+\alpha_{j}^{2}\left(q \lambda_{j}-\lambda_{j}-k\right)^{2}}{\left(\lambda_{j}+k\right)^{2}} .
\end{aligned}
$$

\section{Matrix mean square error comparisons}

Before compare the MMSE of the estimators, we used spectral decomposition of $C$ matrix as $C=Q \Lambda Q^{T}$ where $Q$ is the matrix whose columns consist of the eigenvectors of $C$ and $\Lambda$ is a diagonal matrix in which the diagonal elements are the eigenvalues of $C$. After the spectral decomposition, the MMSE of the estimators can be rewritten as follows

$$
\begin{gathered}
M M S E\left(\widehat{\beta}_{M L}\right)=\tau Q \Lambda^{-1} Q^{T}, \\
M M S E\left(\widehat{\beta}_{I G R E}(k)\right)=\tau Q \Lambda_{k}^{-1} \Lambda \Lambda_{k}^{-1} Q^{T}+b_{I G R E}^{\star} b_{I G R E}^{\star^{T}},
\end{gathered}
$$

where $\Lambda_{k}=(\Lambda+k I)^{-1}$ and $b_{I G R E}^{\star}=-k\left(Q \Lambda Q^{T}+k I\right)^{-1} \beta$.

$$
\operatorname{MMSE}\left(\widehat{\beta}_{I G T P R E}(k, q)\right)=q^{2} \tau Q \Lambda_{k}^{-1} \Lambda \Lambda_{k}^{-1} Q^{T}+b_{I G T P R E}^{\star} b_{I G T P R E}^{T^{T}},
$$

where $b_{I G T P R E}^{\star}=\left[q Q \Lambda_{k}^{-1} \Lambda Q^{T}-I\right] \beta$.

We have to give the following lemmas to compare the proposed estimator with the ML estimator and IGRE.

Lemma 4.1 ([10]). Let $\Delta$ be a positive definite (p.d.) matrix, $\alpha$ be a nonzero vector and, $c$ be a positive constant. Then $c \Delta-\alpha \alpha^{T}>0$ if and only if (iff) $\alpha^{T} \Delta^{-1} \alpha<c$.

Lemma $4.2([23])$. Let $\widehat{\beta}_{1}$ and $\widehat{\beta}_{2}$ are two estimators of the unknown coefficient vector $\beta$. Let $\Omega=\operatorname{Var}\left(\widehat{\beta}_{1}\right)-\operatorname{Var}\left(\widehat{\beta}_{2}\right)$ be p.d. and, $b_{1}=\operatorname{Bias}\left(\widehat{\beta}_{1}\right)$ and $b_{2}=\operatorname{Bias}\left(\widehat{\beta}_{2}\right)$. MMSE $\left(\widehat{\beta}_{1}\right)-$ $\operatorname{MMSE}\left(\widehat{\beta}_{2}\right)>0$ iff $b_{2}^{T}\left(\Omega+b_{1} b_{1}^{T}\right)^{-1} b_{2}<1$.

In the following theorem, the superiority of the IGTPRE over the ML estimator according to the MMSE criteria is shown. 
Theorem 4.3. Let $k>\lambda_{j}(q-1)$ where $j=1, \ldots, p+1$ and $q>1$. MMSE $\left(\widehat{\beta}_{M L}\right)-$ $\operatorname{MMSE}\left(\widehat{\beta}_{\operatorname{IGTPRE}}(k, q)\right)>0$ iff $b_{I G T P R E}^{\star^{T}} Q\left\{\Lambda^{-1}-q^{2} \Lambda_{k}^{-1} \Lambda \Lambda_{k}^{-1}\right\}^{-1} Q^{T} b_{I G T P R E}^{\star}<\tau$.

Proof.

$$
\begin{aligned}
\operatorname{MMSE}\left(\widehat{\beta}_{M L}\right)-\operatorname{MME}\left(\widehat{\beta}_{\text {IGTPRE }}(k, q)\right) & =\tau Q\left[\Lambda^{-1}-q^{2} \Lambda_{k}^{-1} \Lambda \Lambda_{k}^{-1}\right] Q^{T} \\
& -b_{I G T P R E}^{\star} b_{I G T P R E}^{\star_{I}^{T}} \\
& =\tau Q \operatorname{diag}\left\{\frac{1}{\lambda_{j}}-\frac{q^{2} \lambda_{j}}{\left(\lambda_{j}+k\right)^{2}}\right\}_{j=1}^{p+1} Q^{T} \\
& -b_{I G T P R E}^{\star} b_{I G T P R E}^{\star^{T}} .
\end{aligned}
$$

$\left(\Lambda^{-1}-q^{2} \Lambda_{k}^{-1} \Lambda \Lambda_{k}^{-1}\right)$ is positive definite (p.d.) if $k^{2}+2 \lambda_{j} k+\left(1-q^{2}\right) \lambda_{j}^{2}>0$. Thus, if $k>\lambda_{j}(q-1)$ then, $\left(\Lambda^{-1}-q^{2} \Lambda_{k}^{-1} \Lambda \Lambda_{k}^{-1}\right)$ is p.d. It implies that the proof is completed via Lemma 4.1.

To illustrate the superiority of the IGTPRE to IGRE, we extend the theorem from the work of [20].

Theorem 4.4. i) $M M S E\left(\widehat{\beta}_{I G T P R E}(k, q)\right)-M M S E\left(\widehat{\beta}_{I G R E}(k)\right)>0$,

if only $b_{I G R E}^{\star^{T}}\left(\Omega+b_{I G T P R E}^{\star} b_{I G T P R E}^{\star^{T}}\right)^{-1} b_{I G R E}^{\star}<1$,

where $\Omega=\operatorname{Var}\left(\widehat{\beta}_{\text {IGTPRE }}(k, q)\right)-\operatorname{Var}\left(\widehat{\beta}_{\text {IGRE }}(k)\right)$ and $q>1$.

ii) $M M S E\left(\widehat{\beta}_{I G R E}(k)\right)-M M S E\left(\widehat{\beta}_{\text {IGTPRE }}(k, q)\right)>0$,

if only $b_{I G T P R E}^{\star^{T}}\left(\Omega^{\star}+b_{I G R E}^{\star} b_{I G R E}^{\star^{T}}\right) b_{I G T P R E}^{\star}<1$,

where $\Omega^{\star}=\operatorname{Var}\left(\widehat{\beta}_{\text {IGRE }}(k)\right)-\operatorname{Var}\left(\widehat{\beta}_{\operatorname{IGTPRE}}(k, q)\right)$ and $q<1$.

Proof. i)

$$
\begin{aligned}
\Omega & =\operatorname{Var}\left(\widehat{\beta}_{\operatorname{IGTPRE}}(k, q)\right)-\operatorname{Var}\left(\widehat{\beta}_{\text {IGRE }}(k)\right) \\
& =q^{2} \tau Q \Lambda_{k}^{-1} \Lambda \Lambda_{k}^{-1} Q^{T}-\tau Q \Lambda_{k}^{-1} \Lambda \Lambda_{k}^{-1} Q^{T} \\
& =\left(q^{2}-1\right) \tau Q \Lambda_{k}^{-1} \Lambda \Lambda_{k}^{-1} Q^{T} .
\end{aligned}
$$

Due to $k>0$ and $q>1, \Omega$ is a p.d. According to the Lemma 4.2, the proof is completed. ii)

$$
\begin{aligned}
\Omega^{\star} & =\operatorname{Var}\left(\widehat{\beta}_{\text {IGRE }}(k)\right)-\operatorname{Var}\left(\widehat{\beta}_{\operatorname{IGTPRE}}(k, q)\right) \\
& =\tau Q \Lambda_{k}^{-1} \Lambda \Lambda_{k}^{-1} Q^{T}-q^{2} \tau Q \Lambda_{k}^{-1} \Lambda \Lambda_{k}^{-1} Q^{T} \\
& =\left(1-q^{2}\right) \tau Q \Lambda_{k}^{-1} \Lambda \Lambda_{k}^{-1} Q^{T} .
\end{aligned}
$$

When $k>0$ and $q<1$ then $\Omega^{\star}$ is a p.d. Due to the Lemma 4.2 , the proof is completed.

\section{Selection of the parameters $k$ and $q$}

To obtain the optimal values of the $k$ and $q$ parameters, we can take the derivative of the MSE of the IGTPRE given in Eq. (3.7). After taking the derivative concerning $k$ for a fixed value of $q$ then equating numerator to zero, the optimal value of $k$ can be obtained as

$$
\widehat{k}=\frac{q \sum_{j=1}^{p+1} \widehat{\tau} \lambda_{j}+(q-1) \sum_{j=1}^{p+1} \widehat{\alpha}_{j}^{2} \lambda_{j}^{2}}{\sum_{j=1}^{p+1} \widehat{\alpha}_{j}^{2} \lambda_{j}} .
$$


We get the optimal value of $q$ parameter for a fixed value of $k$ as follows

$$
\widehat{q}=\frac{\sum_{j=1}^{p+1} \frac{\widehat{\alpha}_{j}^{2} \lambda_{j}}{\lambda_{j}+k}}{\sum_{j=1}^{p+1} \frac{\widehat{\tau} \lambda_{j}+\widehat{\alpha}_{j}^{2} \lambda_{j}^{2}}{\left(\lambda_{j}+k\right)^{2}}} .
$$

In this paper, we use following methods to choose $k$ and $q$ parameters. This procedures modified from the work of [9]. We called as $I G T P R E_{1}$ if following procedure is used to choose $k$ and $q$ parameters.

Step 1. Choose $k$ parameter as $\widehat{k}>\frac{\widehat{\tau}}{\widehat{\alpha}_{j}^{2}}$.

Step 2. Using the estimated $k$ value obtained in Step 1, compute $q$ parameter as given in Eq. (5.2).

When we compute $k$ and $q$ parameters by means of the following procedure, the proposed estimator is called as IGTPRE $E_{2}$.

Step 1. Compute $q$ parameter as given in Eq. (5.2).

Step 2. Using the $q$ value obtained in Step 1, compute $k$ parameter as

$$
\widehat{k}=\frac{1}{p+1} \sum_{j=1}^{p+1} \frac{\widehat{q} \widehat{\tau} \lambda_{j}+(\widehat{q}-1) \lambda_{j}^{2} \widehat{\alpha}_{j}^{2}}{\lambda_{j} \widehat{\alpha}_{j}^{2}} .
$$

The third procedure modified from the work of [5]. The estimator using the third procedure to calculate $k$ and $q$ parameters is called as IGTPRE $E_{3}$.

Step 1. Compute the initial value of $q$ as

$$
\widehat{q}>\sum_{j=1}^{p+1} \frac{\lambda_{j} \widehat{\alpha}_{j}^{2}}{\widehat{\tau}+\lambda_{j} \widehat{\alpha}_{j}^{2}} .
$$

Step 2. Compute the initial value of $k$ parameter as

$$
\widehat{k}=\frac{\sum_{j=1}^{p+1}\left(\widehat{q} \widehat{\tau} \lambda_{j}+(\widehat{q}-1) \lambda_{j}^{2} \widehat{\alpha}_{j}^{2}\right)}{\sum_{j=1}^{p+1} \lambda_{j} \widehat{\alpha}_{j}^{2}} .
$$

Step 3. Obtain the optimal value of $q$ using Eq. (5.2) with the $k$ parameter obtained in Step 2.

Step 4. Calculate the optimal value of $k$ parameter using the optimal value of $q$ parameter obtained in Step 3.

\section{Monte Carlo simulation study}

In this section, we perform a Monte Carlo simulation to evaluate the superiority of the proposed estimator to the other estimators when multicollinearity with different degrees exist due to the theoretical results are not enough to illustrate the performance of the estimators. Also, we examine the effects of the number of the explanatory variables $p$, dispersion parameter $\tau$, and the sample size $n$.

\subsection{Simulation design}

In the simulation study, we have considered three different correlation values as $\rho=$ $0.90,0.95$ and 0.99. Six different sample size have been chosen as $n=50,100,200,300,400$ 
and 500. $\tau$ values have been taken as $0.5,1.5,3$. The explanatory variables have been generated according to the formula of [15] as

$$
x_{i j}=\left(1-\rho^{2}\right)^{\frac{1}{2}} z_{i j}+\rho z_{i(p+1)},
$$

where $z_{i j}$ 's are pseudo-random numbers generated from standard normal distribution, $i=1,2, \ldots, n$ and $j=1,2, \ldots, p$. Dependent variable is generated from the IG distribution as $y_{i} \sim I G\left(\mu_{i}, \tau\right)$ where $\mu_{i}$ is the mean function such that $\mu_{i}=\frac{1}{\sqrt{x_{i}^{T} \beta}}$. Coefficient vector is chosen using the widespread restriction, $\beta^{T} \beta=1$, that has been used in GLMs by many authors (see, e.g., $[1,3,6]$ ).

Condition number $(\kappa)$, is used to check whether collinearity exist among the explanatory variables or not, is given as

$$
\kappa=\sqrt{\frac{\lambda_{\max }}{\lambda_{\min }}},
$$

where $\lambda_{\max }$ and $\lambda_{\min }$ are the maximum and minimum eigenvalues of the $C$ matrix, respectively. If $\kappa<10$ then there is no multicollinearity problem. There is a moderate multicollinearity if $10<\kappa<30$, If $\kappa>30$ then there is a severe multicollinearity. The simulation has been run for 1000 times. The estimated MSE value, which is given as follows, has been used to compare the estimators' performances.

$$
\operatorname{MSE}(\tilde{\beta})=\frac{1}{1000} \sum_{i=1}^{1000}\left(\tilde{\beta}_{i}-\beta\right)^{T}\left(\tilde{\beta}_{i}-\beta\right),
$$

where $\tilde{\beta}_{i}$ is the estimated coefficient related to the interested estimator in the ith replication.

\subsection{Simulation results and discussion}

In this subsection, simulation results have been discussed. Tables 1-4 present that the estimated MSEs corresponding to the related estimators for the different values of the $\rho$, $n, p$ and $\tau$. When we look into the Tables $1-4$, we can observe the following situations.

If the correlation's degree is increased, the MSEs of the estimators increase for all scenarios. Specifically, the correlation degree has more effect on the MSE values of ML estimator and IGRE for the small sample size. On the other hand, the number of explanatory variables has a negative effect on the MSE values. When the number of explanatory variables increases, the MSE values of the considered estimators increase for the same $\rho$, $n$, and $\tau$ values. We can also see from the Tables 1-4 that when the $\tau$ value is getting bigger, the MSEs are getting bigger. So, the dispersion parameter $\tau$ has a negative effect on the MSE values. The last factor thinking of the simulation scenarios is the sample size. From this point of view, if the sample size increases, then the considered estimators' MSE values decrease. When the estimators compare, the IGTPRE estimators, using different algorithms to select $k$ and $q$ values, have smaller MSE values than the ML estimator and IGRE. Among the three different IGTPRE, IGTPRE $E_{3}$ has the smallest MSE values. As a result of the simulation study, it can be seen that the $I G T P R E_{3}$ estimator is the most robust estimator for different $\rho, n, p$, and $\tau$ values.

\section{A real data example}

In this section, we give a real data example to show the proposed estimator's performance in the application. Stack loss data, which is used firstly by [7], has been used to illustrate the proposed estimator's performance. After, Amin et al. [3] used the data set to show the performance of the new ridge estimators in the inverse Gaussian regression model. In this data set, the dependent variable $(\mathrm{y})$ is the percent of the ingoing ammonia 
that is lost by escaping in the unabsorbed nitric oxides. Independent variables are as follows: $x_{1}=$ air flow (which reflects the plant's operation rate), $x_{2}=$ temperature of the cooling water in the coils of the absorbing tower for the nitric oxides, $x_{3}=$ concentration of nitric acid in the absorbing liquid.

The goodness of fit (GoF) tests are used to examine which distribution is well modeled in the data set. The results of the GoF tests are given in Table 5. We also give the Q-Q plots of the related distributions for the data set to support goodness of fit tests visually in Figure 1.

We investigate whether multicollinearity exists in the data set or not. For this aim, we calculate the condition number. As a result of the $\kappa=56.57735$, there exist severe multicollinearity among the explanatory variables. Therefore, we propose IGTPRE to handle multicollinearity in the IGR model. The estimated regression coefficients of the estimators and MSEs are given in Table 6. From Table 6, it is observed that the performance of the proposed IGTPREs better than the ML estimator and IGRE. As a result of the real data example, IGTPREs can be used as an alternative estimator when the multicollinearity exists in the IGR model.

\section{Conclusions}

In the IGR model, ML estimator is the traditional method to estimate unknown regression coefficients, but if multicollinearity exists among the explanatory variables, the ML estimation produces the wrong sign of coefficient and higher variance than the usual. We propose IGTPRE to overcome the multicollinearity problem. Since the performance of the IGTPRE depends on the selection of the biasing parameter $k$ and q, we propose three different algorithm to obtain these parameters. We conduct a simulation study under different factors to show the performance of the proposed estimator. The results of the simulation study show that IGTPRE is superior to ML estimator and IGRE. Also, we give a real data example to illustrate the performance of the proposed estimator. As a result of a simulation study and real data example, IGTPRE can be used as an alternative biased estimator to combat the effect of a multicollinearity problem.

Acknowledgment. The authors thank the editor, associate editor, and the referees for their constructive comments and suggestions which lead to significant improvements in this article.

\section{References}

[1] M.N. Akram, M. Amin and M. Qasim, A new Liu-type estimator for the inverse Gaussian regression model, J. Stat. Comput. Simul. 90 (7), 1153-1172, 2020.

[2] Z.Y. Algamal, Performance of Ridge estimator in inverse Gaussian regression model, Comm. Statist. Theory Methods 48 (15), 3836-3849, 2019.

[3] M. Amin, M. Qasim, S. Afzal and K. Naveed, New Ridge estimators in the inverse Gaussian regression model: Monte Carlo simulation and application to chemical data, Comm. Statist. Simulation Comput.,doi: 10.1080/03610918.2020.1797794, 2020.

[4] M. Amin, M. Qasim and M. Amanullah, Performance of Asar and Genç and Huang and Yang's two-parameter estimation methods for the gamma regression model, Iran. J. Sci. Technol. Trans. A Sci. 43, 2951-2963, 2019.

[5] Y. Asar, Liu Type Logistic Estimators, PhD thesis, Institute of Science, Selcuk University, Konya, Turkey, 2015.

[6] Y. Asar and A. Genç, Two-parameter Ridge estimator in the binary logistic regression, Comm. Statist. Simulation Comput. 46 (9), 7088-7099, 2017.

[7] K.A. Brownlee, Statistical Theory and Methodology in Science and Engineering, Wiley, New York, 1965. 
[8] R.S. Chhikara and J.L. Folks, The Inverse Gaussian Distribution: Theory, Methodology and Applications, Marcel Dekker, New York, 1989.

[9] H. Ertaş, S. Toker and S. Kaçıranlar,Robust two parameter Ridge M-estimator for linear regression, J. Appl. Stat. 42 (7), 1490-1502, 2015.

[10] R.W. Farebrother, Further results on the mean square error of Ridge regression, J. R. Stat. Soc. Ser. B. Stat. Methodol. 38, 248-250, 1976.

[11] A.E. Hoerl and R.W. Kennard, Ridge regression: biased estimation for nonorthogonal problems, Technometrics 12 (1), 55-67, 1970.

[12] J. Huang and H. Yang, A two-parameter estimator in the negative binomial regression model, Comm. Statist. Simulation Comput. 84 (1), 124-134, 2014.

[13] S. Lipovetsky, Two parameter Ridge regression and its convergence o the eventual pairwise model, Math Comput Model 44, 304-318, 2006.

[14] S. Lipovetsky and W.M. Conklin, Ridge regression in two-parameter solution, Appl. Stoch. Models Bus. Ind. 21 (6), 525-540, 2005.

[15] G.C. McDonald and D.I. Galarneau, A Monte Carlo evaluation of some Ridge-type estimators, J. Amer. Statist. Assoc. 70 (350), 407-416, 1975.

[16] K. Naveed, M. Amin, S. Afzal and M. Qasim, New shrinkage parameters for the inverse Gaussian Liu regression, Comm. Statist. Theory Methods, doi: 10.1080/03610926.2020.1791339, 2020.

[17] M.R. Özkale and S. Kaçıranlar, The restricted and unrestricted two-parameter estimators, Comm. Statist. Theory Methods 36, 2707-2725, 2007.

[18] A. Punzo, A new look at the inverse Gaussian distribution with applications to insurance and economic data, J. Appl. Stat. 46 (7), 1260-1287, 2019.

[19] V. Seshadri, The Inverse Gaussian Distribution: Statistical Theory and Applications, Springer, New York, Volume 137 of Notes in Statistics, 2012.

[20] S. Toker, Investigating the two parameter analysis of Lipovetsky for simultaneous systems, Statist. Papers 61, 2059-2089, 2020.

[21] S. Toker and S. Kaçiranlar, On the performance of two parameter ridge estimator under the mean square error criterion, Appl. Math. Comput. 219, 4718-4728, 2013.

[22] S. Toker, G. Şiray and M.Qasim, Developing a first order two parameter estimator for the generalized linear models, 11th International Statistics Congress (ISC2019), Muğla, Turkey, 2019.

[23] G. Trenkler and H. Toutenburg, Mean squared error matrix comparisons between biased estimators-an overwiev of recent results, Statist. Papers 31, 165-179, 1990.

[24] M.C.K. Tweedie,Statistical properties of inverse Gaussian distributions, I, Ann. Math. Statist. 28 (2), 362-377, 1957 
Table 1. The estimated MSE values $(p=2)$.

\begin{tabular}{|c|c|c|c|c|c|c|c|}
\hline$n$ & $\tau$ & $\rho$ & ML & $I G R E$ & $I G T P R E_{1}$ & $I G T P R E_{2}$ & $I G T P R E_{3}$ \\
\hline \multirow{9}{*}{50} & \multirow[t]{3}{*}{0.5} & 0.90 & 5.98629 & 3.70038 & 1.54788 & 0.19922 & 0.03096 \\
\hline & & 0.95 & 11.35896 & 6.58318 & 2.74488 & 0.22776 & 0.03940 \\
\hline & & 0.99 & 56.47398 & 30.80135 & 3.64370 & 0.25699 & 0.04845 \\
\hline & \multirow[t]{3}{*}{1.5} & 0.90 & 19.84681 & 12.05575 & 4.04066 & 0.32600 & 0.11792 \\
\hline & & 0.95 & 30.39964 & 16.42961 & 4.76981 & 0.39516 & 0.17938 \\
\hline & & 0.99 & 178.77552 & 105.11707 & 8.09853 & 0.50445 & 0.20178 \\
\hline & \multirow[t]{3}{*}{3} & 0.90 & 48.19672 & 27.84578 & 7.34593 & 0.52784 & 0.33724 \\
\hline & & 0.95 & 76.28352 & 42.60114 & 7.56314 & 0.61971 & 0.36862 \\
\hline & & 0.99 & 401.62587 & 214.02481 & 8.47143 & 0.70919 & 0.40705 \\
\hline \multirow{9}{*}{100} & \multirow[t]{3}{*}{0.5} & 0.90 & 3.11363 & 2.00865 & 1.05216 & 0.14273 & 0.02266 \\
\hline & & 0.95 & 7.22839 & 4.48228 & 1.77030 & 0.17051 & 0.02340 \\
\hline & & 0.99 & 29.83047 & 16.29375 & 1.89220 & 0.20005 & 0.03468 \\
\hline & \multirow[t]{3}{*}{1.5} & 0.90 & 8.99316 & 5.11642 & 2.94056 & 0.26600 & 0.05577 \\
\hline & & 0.95 & 16.87495 & 8.59175 & 3.59090 & 0.31357 & 0.07662 \\
\hline & & 0.99 & 86.76842 & 47.97432 & 6.00048 & 0.32329 & 0.08226 \\
\hline & \multirow[t]{3}{*}{3} & 0.90 & 18.45930 & 11.02750 & 4.52226 & 0.31101 & 0.14308 \\
\hline & & 0.95 & 38.59899 & 22.67605 & 5.92591 & 0.36947 & 0.18229 \\
\hline & & 0.99 & 159.22118 & 78.95461 & 6.96286 & 0.54093 & 0.20637 \\
\hline \multirow{9}{*}{200} & \multirow[t]{3}{*}{0.5} & 0.90 & 1.57855 & 1.01465 & 0.40568 & 0.09335 & 0.00958 \\
\hline & & 0.95 & 2.35035 & 1.36323 & 0.51330 & 0.09870 & 0.01479 \\
\hline & & 0.99 & 14.77909 & 8.34481 & 0.95890 & 0.13425 & 0.02273 \\
\hline & \multirow[t]{3}{*}{1.5} & 0.90 & 4.15453 & 2.40137 & 1.45047 & 0.17893 & 0.03013 \\
\hline & & 0.95 & 8.95960 & 5.26754 & 2.48260 & 0.19724 & 0.03483 \\
\hline & & 0.99 & 48.58891 & 26.15763 & 5.70606 & 0.28898 & 0.03972 \\
\hline & \multirow[t]{3}{*}{3} & 0.90 & 8.99012 & 5.49949 & 2.70266 & 0.28152 & 0.06350 \\
\hline & & 0.95 & 17.41016 & 10.43612 & 3.82738 & 0.29591 & 0.06840 \\
\hline & & 0.99 & 89.95973 & 50.13863 & 5.76860 & 0.35063 & 0.10362 \\
\hline \multirow{9}{*}{300} & \multirow[t]{3}{*}{0.5} & 0.90 & 0.88744 & 0.60556 & 0.19185 & 0.08243 & 0.00755 \\
\hline & & 0.95 & 1.81130 & 1.10758 & 0.38504 & 0.08949 & 0.01129 \\
\hline & & 0.99 & 9.73288 & 5.70295 & 0.67113 & 0.10939 & 0.01981 \\
\hline & \multirow[t]{3}{*}{1.5} & 0.90 & 3.05014 & 1.79211 & 0.91618 & 0.15476 & 0.02056 \\
\hline & & 0.95 & 5.73624 & 3.32338 & 1.51862 & 0.17087 & 0.02815 \\
\hline & & 0.99 & 30.14335 & 17.91796 & 3.60323 & 0.22532 & 0.03046 \\
\hline & \multirow[t]{3}{*}{3} & 0.90 & 5.94173 & 3.64484 & 1.72168 & 0.18256 & 0.03135 \\
\hline & & 0.95 & 10.65858 & 6.23272 & 2.48165 & 0.20209 & 0.03834 \\
\hline & & 0.99 & 50.67120 & 27.21082 & 4.84193 & 0.26463 & 0.05427 \\
\hline \multirow{9}{*}{400} & \multirow[t]{3}{*}{0.5} & 0.90 & 0.73119 & 0.51074 & 0.12807 & 0.05745 & 0.00613 \\
\hline & & 0.95 & 1.43619 & 1.00629 & 0.29578 & 0.07774 & 0.00938 \\
\hline & & 0.99 & 6.08607 & 3.17863 & 0.61248 & 0.09757 & 0.01968 \\
\hline & 1.5 & 0.90 & 2.37600 & 1.55340 & 0.74069 & 0.13435 & 0.01401 \\
\hline & & 0.95 & 4.30813 & 2.52015 & 0.81767 & 0.14870 & 0.01839 \\
\hline & & 0.99 & 22.59794 & 13.11079 & 3.40307 & 0.20805 & 0.02701 \\
\hline & 3 & 0.90 & 3.88951 & 2.10620 & 1.06341 & 0.17968 & 0.02917 \\
\hline & & 0.95 & 8.89791 & 4.83462 & 1.93259 & 0.18759 & 0.03150 \\
\hline & & 0.99 & 42.27636 & 23.33666 & 4.04919 & 0.26355 & 0.04884 \\
\hline & 0.5 & 0.90 & 0.52531 & 0.38484 & 0.10880 & 0.05524 & 0.00575 \\
\hline & & 0.95 & 1.56750 & 0.91139 & 0.26953 & 0.06763 & 0.00910 \\
\hline & & 0.99 & 5.28962 & 2.86511 & 0.32982 & 0.07429 & 0.01921 \\
\hline & 1.5 & 0.90 & 1.90443 & 1.25586 & 0.32431 & 0.10243 & 0.01002 \\
\hline 500 & & 0.95 & 3.26897 & 1.98114 & 0.42751 & 0.11786 & 0.01393 \\
\hline & & 0.99 & 18.84272 & 11.25817 & 1.32244 & 0.18719 & 0.02461 \\
\hline & 3 & 0.90 & 3.32326 & 1.93362 & 0.77126 & 0.14443 & 0.02534 \\
\hline & & 0.95 & 7.37695 & 4.37042 & 1.81279 & 0.17154 & 0.02757 \\
\hline & & 0.99 & 31.56758 & 17.03204 & 3.57125 & 0.20781 & 0.04177 \\
\hline
\end{tabular}


Table 2. The estimated MSE values $(p=4)$.

\begin{tabular}{|c|c|c|c|c|c|c|c|}
\hline$n$ & $\tau$ & $\rho$ & ML & $I G R E$ & $I G T P R E_{1}$ & $I G T P R E_{2}$ & $I G T P R E_{3}$ \\
\hline \multirow{9}{*}{50} & \multirow[t]{3}{*}{0.5} & 0.90 & 23.15681 & 13.69545 & 5.35790 & 0.40504 & 0.03560 \\
\hline & & 0.95 & 35.19113 & 17.99411 & 5.43399 & 0.49357 & 0.04393 \\
\hline & & 0.99 & 193.71076 & 104.81346 & 12.73438 & 0.77435 & 0.05491 \\
\hline & \multirow[t]{3}{*}{1.5} & 0.90 & 65.60160 & 37.73343 & 11.13694 & 0.68979 & 0.15538 \\
\hline & & 0.95 & 111.38265 & 59.04244 & 12.59880 & 0.75310 & 0.18351 \\
\hline & & 0.99 & 538.72336 & 260.32122 & 16.11375 & 1.14741 & 0.20875 \\
\hline & \multirow[t]{3}{*}{3} & 0.90 & 122.38956 & 64.98879 & 11.47747 & 0.99222 & 0.34680 \\
\hline & & 0.95 & 300.82297 & 170.77422 & 13.74892 & 1.06554 & 0.39846 \\
\hline & & 0.99 & 1652.41819 & 827.43822 & 18.90552 & 1.26557 & 0.43993 \\
\hline \multirow{9}{*}{100} & \multirow[t]{3}{*}{0.5} & 0.90 & 10.66519 & 6.49061 & 1.53900 & 0.23262 & 0.02013 \\
\hline & & 0.95 & 19.73465 & 11.13333 & 3.05922 & 0.34137 & 0.03022 \\
\hline & & 0.99 & 78.45008 & 37.90870 & 5.32830 & 0.45384 & 0.04049 \\
\hline & \multirow[t]{3}{*}{1.5} & 0.90 & 25.68495 & 14.07548 & 5.90151 & 0.39339 & 0.06825 \\
\hline & & 0.95 & 69.56064 & 43.01715 & 10.86433 & 0.62111 & 0.07925 \\
\hline & & 0.99 & 253.46515 & 135.34917 & 15.69648 & 0.79775 & 0.09170 \\
\hline & \multirow[t]{3}{*}{3} & 0.90 & 52.68490 & 30.03321 & 7.25478 & 0.43626 & 0.17398 \\
\hline & & 0.95 & 107.89926 & 65.16975 & 11.10688 & 0.64160 & 0.19600 \\
\hline & & 0.99 & 478.85707 & 264.22939 & 16.44276 & 0.96330 & 0.21600 \\
\hline \multirow{9}{*}{200} & \multirow[t]{3}{*}{0.5} & 0.90 & 5.12988 & 3.13558 & 0.88140 & 0.17444 & 0.01449 \\
\hline & & 0.95 & 11.75152 & 7.11333 & 1.68920 & 0.22168 & 0.01774 \\
\hline & & 0.99 & 40.88033 & 20.41324 & 2.14678 & 0.32179 & 0.03368 \\
\hline & \multirow[t]{3}{*}{1.5} & 0.90 & 14.43237 & 8.60928 & 3.57115 & 0.30519 & 0.03418 \\
\hline & & 0.95 & 29.34524 & 17.09810 & 5.86406 & 0.44567 & 0.04610 \\
\hline & & 0.99 & 131.86586 & 73.82636 & 14.78229 & 0.72057 & 0.05406 \\
\hline & \multirow[t]{3}{*}{3} & 0.90 & 28.35992 & 17.34592 & 6.96162 & 0.41501 & 0.08739 \\
\hline & & 0.95 & 51.04998 & 29.48653 & 9.00692 & 0.57037 & 0.11848 \\
\hline & & 0.99 & 229.48214 & 113.55335 & 15.24814 & 0.91027 & 0.12219 \\
\hline \multirow{9}{*}{300} & \multirow[t]{3}{*}{0.5} & 0.90 & 3.02686 & 1.84533 & 0.49710 & 0.11937 & 0.01224 \\
\hline & & 0.95 & 5.74714 & 3.39360 & 0.70503 & 0.15266 & 0.01715 \\
\hline & & 0.99 & 26.67726 & 13.46510 & 2.07966 & 0.28089 & 0.03048 \\
\hline & \multirow[t]{3}{*}{1.5} & 0.90 & 10.14137 & 6.11223 & 2.53250 & 0.24172 & 0.02066 \\
\hline & & 0.95 & 18.95917 & 11.09595 & 2.78769 & 0.29802 & 0.02978 \\
\hline & & 0.99 & 93.90571 & 53.84122 & 7.67901 & 0.59845 & 0.04700 \\
\hline & \multirow[t]{3}{*}{3} & 0.90 & 18.71941 & 11.26964 & 3.93846 & 0.38093 & 0.06125 \\
\hline & & 0.95 & 32.89710 & 17.79871 & 6.48260 & 0.51985 & 0.06337 \\
\hline & & 0.99 & 179.72086 & 105.01754 & 13.08728 & 0.79323 & 0.07783 \\
\hline \multirow{9}{*}{400} & \multirow[t]{3}{*}{0.5} & 0.90 & 2.43469 & 1.56117 & 0.35026 & 0.10923 & 0.01137 \\
\hline & & 0.95 & 5.10123 & 3.06810 & 0.53253 & 0.12231 & 0.01589 \\
\hline & & 0.99 & 21.36718 & 11.38033 & 1.47528 & 0.23931 & 0.02930 \\
\hline & 1.5 & 0.90 & 7.39338 & 4.34212 & 1.44969 & 0.20175 & 0.01838 \\
\hline & & 0.95 & 13.79118 & 7.68544 & 1.90683 & 0.21458 & 0.02201 \\
\hline & & 0.99 & 66.96924 & 36.52436 & 6.94737 & 0.55302 & 0.03496 \\
\hline & 3 & 0.90 & 14.75060 & 8.70253 & 3.74040 & 0.31951 & 0.05255 \\
\hline & & 0.95 & 27.66167 & 16.01543 & 5.34610 & 0.38098 & 0.05685 \\
\hline & & 0.99 & 135.65967 & 76.97272 & 11.96248 & 0.57158 & 0.06392 \\
\hline & 0.5 & 0.90 & 1.89075 & 1.21048 & 0.21160 & 0.09404 & 0.00960 \\
\hline & & 0.95 & 3.68760 & 2.14038 & 0.33324 & 0.11079 & 0.01451 \\
\hline & & 0.99 & 19.13865 & 10.39298 & 0.88004 & 0.20032 & 0.02860 \\
\hline & 1.5 & 0.90 & 6.38056 & 3.78749 & 1.18028 & 0.19737 & 0.01694 \\
\hline 500 & & 0.95 & 9.94868 & 5.32465 & 1.33447 & 0.20827 & 0.02183 \\
\hline & & 0.99 & 49.91779 & 26.52025 & 5.35238 & 0.48404 & 0.03390 \\
\hline & 3 & 0.90 & 11.71675 & 7.04216 & 2.26249 & 0.23737 & 0.04240 \\
\hline & & 0.95 & 18.68835 & 10.80053 & 3.75305 & 0.33164 & 0.04835 \\
\hline & & 0.99 & 91.35741 & 50.57125 & 9.40835 & 0.52915 & 0.04970 \\
\hline
\end{tabular}


Table 3. The estimated MSE values $(p=6)$.

\begin{tabular}{|c|c|c|c|c|c|c|c|}
\hline$n$ & $\tau$ & $\rho$ & ML & $I G R E$ & $I G T P R E_{1}$ & $I G T P R E_{2}$ & $I G T P R E_{3}$ \\
\hline \multirow{9}{*}{50} & \multirow[t]{3}{*}{0.5} & 0.90 & 49.79598 & 29.22774 & 8.63955 & 0.64962 & 0.04051 \\
\hline & & 0.95 & 74.80345 & 38.52066 & 9.33076 & 0.92745 & 0.05299 \\
\hline & & 0.99 & 407.71408 & 208.82259 & 20.34138 & 1.20462 & 0.06780 \\
\hline & \multirow[t]{3}{*}{1.5} & 0.90 & 152.48631 & 84.48023 & 17.67229 & 0.93581 & 0.17031 \\
\hline & & 0.95 & 269.09674 & 140.64776 & 19.55157 & 1.00467 & 0.17263 \\
\hline & & 0.99 & 1302.30936 & 674.02037 & 24.33542 & 1.36484 & 0.18521 \\
\hline & \multirow[t]{3}{*}{3} & 0.90 & 332.87019 & 173.22230 & 18.87784 & 1.12511 & 0.37617 \\
\hline & & 0.95 & 670.47943 & 349.39384 & 22.59326 & 1.59833 & 0.40405 \\
\hline & & 0.99 & 3174.83232 & 1571.76607 & 25.95828 & 1.61248 & 0.43554 \\
\hline \multirow{9}{*}{100} & \multirow[t]{3}{*}{0.5} & 0.90 & 20.61677 & 12.09314 & 3.35110 & 0.43658 & 0.02602 \\
\hline & & 0.95 & 33.43757 & 17.85198 & 4.20616 & 0.46225 & 0.02891 \\
\hline & & 0.99 & 171.76425 & 87.72480 & 14.44518 & 1.12392 & 0.04871 \\
\hline & \multirow[t]{3}{*}{1.5} & 0.90 & 59.32922 & 33.10901 & 10.14926 & 0.60125 & 0.06590 \\
\hline & & 0.95 & 109.79152 & 63.49910 & 16.75103 & 0.90242 & 0.08688 \\
\hline & & 0.99 & 424.37234 & 225.03207 & 21.31117 & 1.18397 & 0.09460 \\
\hline & \multirow[t]{3}{*}{3} & 0.90 & 107.25859 & 58.44609 & 12.59657 & 0.78076 & 0.20759 \\
\hline & & 0.95 & 193.49550 & 104.78034 & 17.90481 & 0.93879 & 0.24304 \\
\hline & & 0.99 & 983.64719 & 516.06245 & 24.53250 & 1.33735 & 0.25074 \\
\hline \multirow{9}{*}{200} & \multirow[t]{3}{*}{0.5} & 0.90 & 11.56685 & 7.26040 & 1.75651 & 0.31301 & 0.01397 \\
\hline & & 0.95 & 17.02905 & 9.30904 & 1.85296 & 0.35450 & 0.02184 \\
\hline & & 0.99 & 78.39612 & 41.57001 & 7.01482 & 0.75819 & 0.03871 \\
\hline & \multirow[t]{3}{*}{1.5} & 0.90 & 25.15299 & 14.36482 & 4.96705 & 0.55267 & 0.03770 \\
\hline & & 0.95 & 43.49528 & 23.22584 & 5.38158 & 0.58715 & 0.05175 \\
\hline & & 0.99 & 182.22502 & 91.70383 & 15.43623 & 0.98791 & 0.06392 \\
\hline & \multirow[t]{3}{*}{3} & 0.90 & 52.74452 & 29.17936 & 8.41394 & 0.60246 & 0.10528 \\
\hline & & 0.95 & 90.43823 & 50.40672 & 11.68844 & 0.66458 & 0.11205 \\
\hline & & 0.99 & 413.33351 & 217.59991 & 24.97135 & 1.29971 & 0.12748 \\
\hline \multirow{9}{*}{300} & \multirow[t]{3}{*}{0.5} & 0.90 & 6.04871 & 3.61697 & 0.68271 & 0.19303 & 0.01370 \\
\hline & & 0.95 & 10.67787 & 6.21388 & 1.61627 & 0.27158 & 0.01985 \\
\hline & & 0.99 & 55.14968 & 30.26746 & 5.04186 & 0.63871 & 0.03720 \\
\hline & \multirow[t]{3}{*}{1.5} & 0.90 & 18.29031 & 11.25758 & 4.75335 & 0.42918 & 0.02462 \\
\hline & & 0.95 & 33.02940 & 18.62530 & 5.04281 & 0.54969 & 0.03340 \\
\hline & & 0.99 & 144.85562 & 78.70218 & 15.30966 & 0.89659 & 0.05149 \\
\hline & \multirow[t]{3}{*}{3} & 0.90 & 31.85771 & 17.75132 & 5.31096 & 0.47593 & 0.06573 \\
\hline & & 0.95 & 58.99776 & 31.93741 & 8.46841 & 0.60180 & 0.07455 \\
\hline & & 0.99 & 289.01189 & 158.41676 & 22.26736 & 1.17298 & 0.12724 \\
\hline \multirow{9}{*}{400} & \multirow[t]{3}{*}{0.5} & 0.90 & 5.48456 & 3.39870 & 0.51210 & 0.17259 & 0.01249 \\
\hline & & 0.95 & 9.86340 & 5.56463 & 1.47377 & 0.24158 & 0.01816 \\
\hline & & 0.99 & 41.93431 & 22.77024 & 2.97196 & 0.48749 & 0.03550 \\
\hline & 1.5 & 0.90 & 12.63248 & 7.26501 & 2.23097 & 0.35564 & 0.02072 \\
\hline & & 0.95 & 21.20911 & 11.77835 & 3.70801 & 0.41088 & 0.03070 \\
\hline & & 0.99 & 115.69884 & 64.08244 & 11.30564 & 0.78944 & 0.04208 \\
\hline & 3 & 0.90 & 25.20887 & 14.80232 & 5.57427 & 0.46468 & 0.06065 \\
\hline & & 0.95 & 44.74435 & 24.53422 & 7.10009 & 0.52972 & 0.06265 \\
\hline & & 0.99 & 223.24841 & 122.33871 & 20.08589 & 1.15044 & 0.08861 \\
\hline & 0.5 & 0.90 & 3.69806 & 2.24137 & 0.27672 & 0.13062 & 0.01115 \\
\hline & & 0.95 & 8.47918 & 5.09375 & 1.00870 & 0.20714 & 0.01696 \\
\hline & & 0.99 & 32.91783 & 17.74532 & 2.31285 & 0.42881 & 0.03527 \\
\hline & 1.5 & 0.90 & 11.37701 & 6.46578 & 1.39775 & 0.31566 & 0.01593 \\
\hline 500 & & 0.95 & 18.81489 & 10.13461 & 2.25895 & 0.37949 & 0.02886 \\
\hline & & 0.99 & 79.95187 & 40.81137 & 5.67491 & 0.70167 & 0.03959 \\
\hline & 3 & 0.90 & 21.46639 & 12.45308 & 3.21529 & 0.45838 & 0.03523 \\
\hline & & 0.95 & 37.29451 & 21.54060 & 6.23515 & 0.49550 & 0.05942 \\
\hline & & 0.99 & 164.97241 & 86.45760 & 13.97628 & 0.83610 & 0.07908 \\
\hline
\end{tabular}


Table 4. The estimated MSE values $(p=8)$.

\begin{tabular}{|c|c|c|c|c|c|c|c|}
\hline$n$ & $\tau$ & $\rho$ & ML & $I G R E$ & $I G T P R E_{1}$ & $I G T P R E_{2}$ & $I G T P R E_{3}$ \\
\hline \multirow{9}{*}{50} & \multirow[t]{3}{*}{0.5} & 0.90 & 82.81962 & 45.07752 & 11.57014 & 1.05048 & 0.03819 \\
\hline & & 0.95 & 156.68463 & 85.08876 & 17.77117 & 1.26653 & 0.04000 \\
\hline & & 0.99 & 555.29280 & 259.46267 & 29.67823 & 2.04583 & 0.06120 \\
\hline & \multirow[t]{3}{*}{1.5} & 0.90 & 245.89716 & 125.23176 & 19.26807 & 1.09223 & 0.10481 \\
\hline & & 0.95 & 390.80910 & 178.78677 & 22.17557 & 1.37610 & 0.17247 \\
\hline & & 0.99 & 1792.79214 & 786.09564 & 31.19136 & 2.14476 & 0.19572 \\
\hline & \multirow[t]{3}{*}{3} & 0.90 & 583.03382 & 272.82910 & 20.41452 & 1.15030 & 0.39537 \\
\hline & & 0.95 & 1157.13579 & 545.45901 & 23.82373 & 1.56169 & 0.42007 \\
\hline & & 0.99 & 5176.52937 & 2341.36552 & 36.73224 & 2.39138 & 0.45252 \\
\hline \multirow{9}{*}{100} & \multirow[t]{3}{*}{0.5} & 0.90 & 34.32325 & 19.17733 & 4.82249 & 0.68724 & 0.02050 \\
\hline & & 0.95 & 65.57366 & 36.83444 & 10.00424 & 0.98611 & 0.03114 \\
\hline & & 0.99 & 255.76234 & 130.35422 & 19.23227 & 1.68277 & 0.04356 \\
\hline & \multirow[t]{3}{*}{1.5} & 0.90 & 96.00388 & 53.51968 & 13.37827 & 0.90522 & 0.08326 \\
\hline & & 0.95 & 136.85603 & 70.94898 & 16.78044 & 1.15717 & 0.10263 \\
\hline & & 0.99 & 708.89460 & 356.72625 & 28.61825 & 1.77178 & 0.11324 \\
\hline & \multirow[t]{3}{*}{3} & 0.90 & 184.15767 & 100.82406 & 13.91206 & 0.92311 & 0.24589 \\
\hline & & 0.95 & 345.38649 & 177.12919 & 22.33658 & 1.24591 & 0.25078 \\
\hline & & 0.99 & 1502.21805 & 688.79396 & 30.59780 & 1.85047 & 0.25808 \\
\hline \multirow{9}{*}{200} & \multirow[t]{3}{*}{0.5} & 0.90 & 16.44558 & 9.11408 & 1.78397 & 0.40855 & 0.01600 \\
\hline & & 0.95 & 29.04236 & 15.82474 & 2.48904 & 0.56082 & 0.02320 \\
\hline & & 0.99 & 133.39169 & 69.66032 & 9.17112 & 1.20909 & 0.04006 \\
\hline & \multirow[t]{3}{*}{1.5} & 0.90 & 44.78968 & 25.54793 & 7.10898 & 0.74963 & 0.04386 \\
\hline & & 0.95 & 79.08911 & 43.91222 & 9.82067 & 0.87898 & 0.05196 \\
\hline & & 0.99 & 323.42888 & 167.74340 & 26.02317 & 1.74865 & 0.05967 \\
\hline & \multirow[t]{3}{*}{3} & 0.90 & 78.98286 & 44.41149 & 12.30768 & 0.80771 & 0.13206 \\
\hline & & 0.95 & 153.73459 & 82.75249 & 14.30956 & 1.00168 & 0.14016 \\
\hline & & 0.99 & 641.41467 & 336.89301 & 29.35917 & 1.79995 & 0.15555 \\
\hline \multirow{9}{*}{300} & \multirow[t]{3}{*}{0.5} & 0.90 & 10.25618 & 5.75760 & 1.03933 & 0.29645 & 0.01387 \\
\hline & & 0.95 & 18.84530 & 10.60295 & 1.90514 & 0.44487 & 0.02093 \\
\hline & & 0.99 & 70.91805 & 35.89461 & 5.97383 & 0.85731 & 0.03905 \\
\hline & \multirow[t]{3}{*}{1.5} & 0.90 & 27.59083 & 15.64417 & 5.30942 & 0.60423 & 0.02728 \\
\hline & & 0.95 & 50.54837 & 27.97628 & 7.12285 & 0.78762 & 0.03473 \\
\hline & & 0.99 & 238.40274 & 127.46732 & 25.11628 & 1.58266 & 0.04552 \\
\hline & \multirow[t]{3}{*}{3} & 0.90 & 51.76425 & 29.21042 & 8.42434 & 0.75232 & 0.09204 \\
\hline & & 0.95 & 101.30284 & 55.98286 & 13.59812 & 0.89637 & 0.10296 \\
\hline & & 0.99 & 435.22702 & 222.51725 & 28.38425 & 1.63339 & 0.10525 \\
\hline \multirow{9}{*}{400} & \multirow[t]{3}{*}{0.5} & 0.90 & 8.85298 & 5.41353 & 0.75683 & 0.28774 & 0.01289 \\
\hline & & 0.95 & 12.61064 & 6.98998 & 1.18838 & 0.29943 & 0.01938 \\
\hline & & 0.99 & 53.41438 & 26.22132 & 4.09785 & 0.67900 & 0.03830 \\
\hline & 1.5 & 0.90 & 23.39380 & 13.38862 & 2.44744 & 0.53294 & 0.01951 \\
\hline & & 0.95 & 41.82386 & 23.72713 & 4.86459 & 0.66542 & 0.02753 \\
\hline & & 0.99 & 163.75601 & 86.85036 & 17.55716 & 1.43915 & 0.04280 \\
\hline & 3 & 0.90 & 38.97308 & 22.32604 & 6.37909 & 0.67061 & 0.06724 \\
\hline & & 0.95 & 67.91877 & 39.11774 & 9.10274 & 0.83051 & 0.07231 \\
\hline & & 0.99 & 311.05535 & 162.46929 & 26.26625 & 1.60003 & 0.09044 \\
\hline & 0.5 & 0.90 & 6.86611 & 4.12757 & 0.56247 & 0.22054 & 0.01236 \\
\hline & & 0.95 & 9.72580 & 5.49079 & 0.76622 & 0.24221 & 0.01872 \\
\hline & & 0.99 & 43.19890 & 25.24120 & 2.88648 & 0.65287 & 0.03582 \\
\hline & 1.5 & 0.90 & 17.59021 & 10.14313 & 2.22224 & 0.48037 & 0.01856 \\
\hline 500 & & 0.95 & 32.26104 & 18.12232 & 3.91271 & 0.57379 & 0.02245 \\
\hline & & 0.99 & 122.21553 & 63.87107 & 15.64739 & 1.35923 & 0.04008 \\
\hline & 3 & 0.90 & 31.78770 & 18.52693 & 5.30794 & 0.61891 & 0.04750 \\
\hline & & 0.95 & 59.74703 & 33.05187 & 8.32714 & 0.74662 & 0.05824 \\
\hline & & 0.99 & 220.91754 & 113.12062 & 21.12842 & 1.44913 & 0.07629 \\
\hline
\end{tabular}




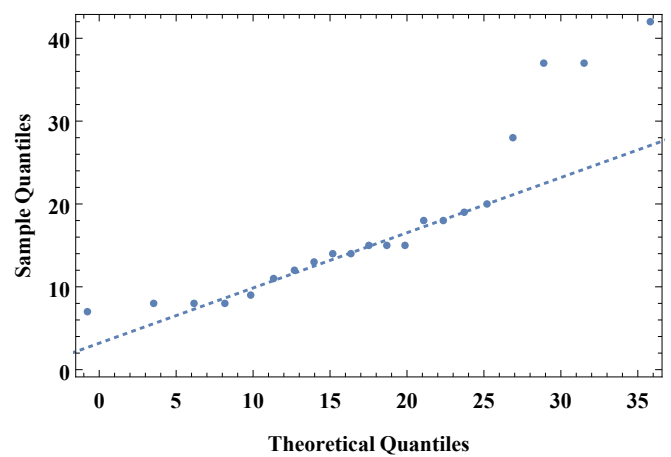

(a) Normal Q-Q Plot

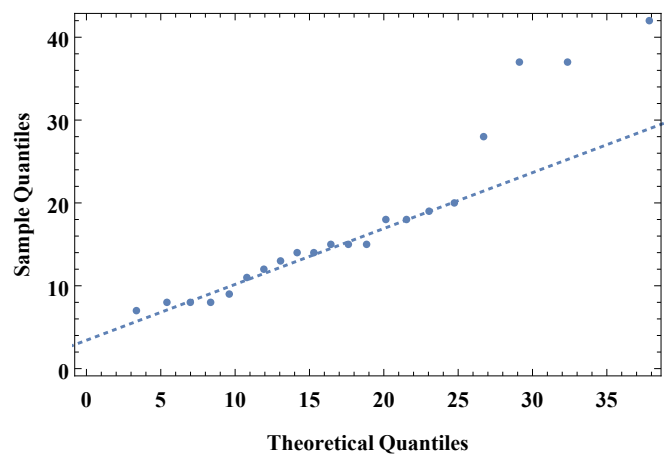

(c) Weibull Q-Q Plot

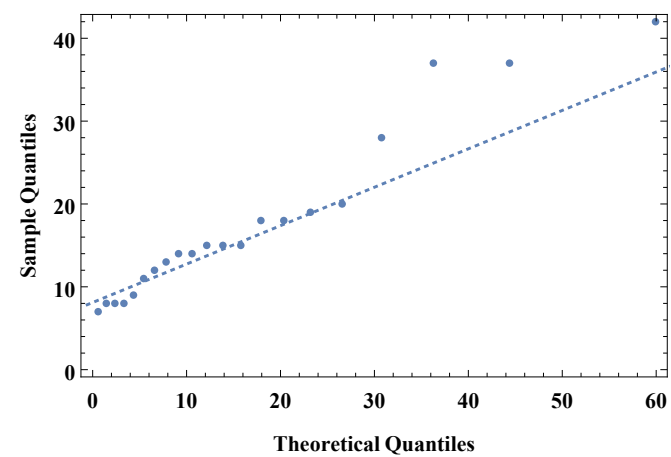

(b) Exponential Q-Q Plot

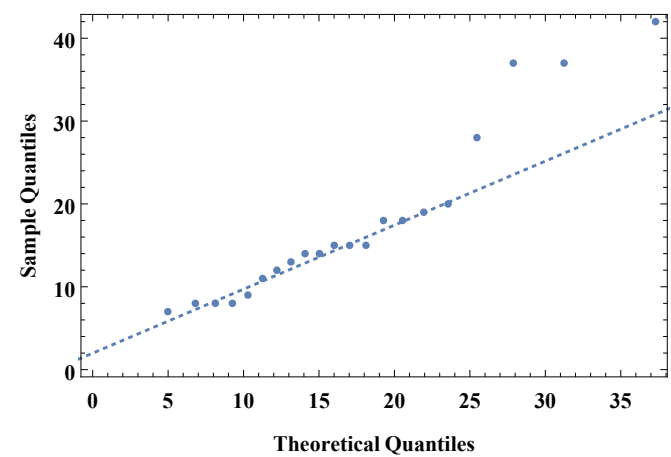

(d) Gamma Q-Q Plot

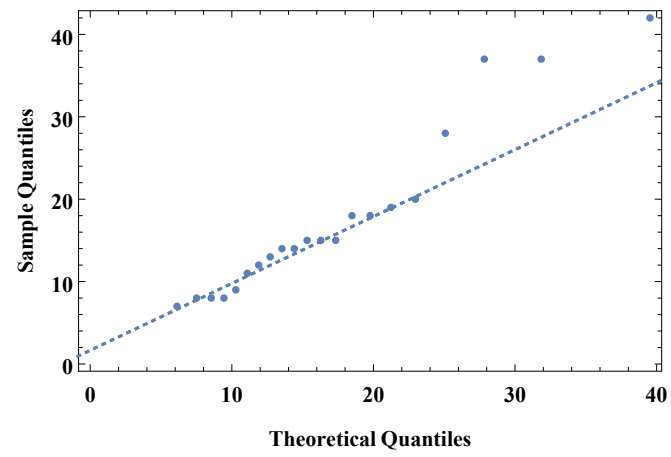

(e) Inverse Gaussian Q-Q Plot

Figure 1. Quantile functions of the distributions 
Table 5. Goodness of fit tests

\begin{tabular}{lrccccc}
\hline & & \multicolumn{5}{c}{ Probability Distributions } \\
\cline { 3 - 7 } Goodness of fit tests & & Normal & Exponential & Weibull & Gamma & IG \\
\hline \multirow{2}{*}{ Anderson-Darling } & Statistic & 1.41573 & 2.57771 & 0.87214 & 0.67514 & 0.44863 \\
& p-value & 0.00100 & 0.00230 & 0.02407 & 0.07814 & 0.39344 \\
\hline \multirow{2}{*}{ Cramer-von Mises } & Statistic & 0.23629 & 0.48985 & 0.13936 & 0.09921 & 0.06132 \\
& p-value & 0.00166 & 0.00123 & 0.02955 & 0.11509 & 0.51472 \\
\hline \multirow{2}{*}{ Pearson $\chi^{2}$} & Statistic & 14.00000 & 15.33333 & 6.00000 & 4.66667 & 6.00000 \\
& p-value & 0.00729 & 0.00903 & 0.19915 & 0.32324 & 0.19915 \\
\hline
\end{tabular}

Table 6. Estimated coefficients and MSEs of the estimators for stack loss data.

\begin{tabular}{lccccc}
\hline Estimators & $\beta_{0}$ & $\beta_{1}$ & $\beta_{2}$ & $\beta_{3}$ & MSE \\
\hline$M L$ & -1.14339 & 0.04591 & 0.05085 & 0.00057 & 0.31933 \\
$I G R E$ & -0.66118 & 0.04682 & 0.04625 & -0.00461 & 0.18488 \\
$I G T P R E_{1}$ & -0.00347 & 0.08338 & 0.05117 & -0.01527 & 0.00462 \\
$I G T P R E_{2}$ & 0.00033 & 0.02051 & 0.00719 & 0.02871 & 0.00075 \\
$I G T P R E_{3}$ & 0.00021 & 0.01243 & 0.00436 & 0.01783 & 0.00003 \\
\hline
\end{tabular}

